\title{
Comparing the ease of identifying the subarachnoid space at midline of L3 -L4 interspace by preprocedural ultrasound versus landmark technique - A randomized control study
}

\author{
Bhavani Vaidiyanathan ${ }^{1}$, Sangeeta Dhanger ${ }^{2, *}$, Joseph Raajesh $\mathbf{I}^{3}$ \\ ${ }^{1,2}$ Assistant Professor, ${ }^{3}$ Professor and HOD, Dept. of Anaesthesia and Critical Care, Indira Gandhi Medical College \& Research \\ Institute, Puducherry, India
}

*Corresponding Author: Sangeeta Dhanger

Email: drsangeeta06@gmail.com

Received: $22^{\text {nd }}$ June, 2018

Accepted: $28^{\text {th }}$ August, 2018

\begin{abstract}
Introduction: The role of ultrasound in central neuraxial blockade has been underappreciated, partly because of the relative ease of the landmark-guided technique and falsely perceived difficulty in imaging through the narrow acoustic windows. This study was designed to compare conventional landmark technique with preprocedural ultrasonography for identification of the subarachnoid space in patients for elective surgeries under spinal anaesthesia.

Materials and Methods: Total 120 patients who were scheduled for elective surgery under spinal anaesthesia were included in this prospective randomised control trial and divided into 2 group, Group L $(n=60)$ (landmark guided technique) and Group U (n $=60$ ) (ultrasound guided technique). Parameters such as time taken for the identification of the interspace, number of insertion attempts (the primary outcome), number of passes and time taken were recorded in both the groups.

Result: The time taken for identification of interspinous space was significantly more in Group U (4.7 $\pm 2.1 \mathrm{mins})$ as compared to Group L $(1.17 \pm 0.52 \mathrm{~min})$ but the number of attempts for needle insertion $(1.12 \pm 0.41 \mathrm{vs} .1 .86 \pm 0.91)$, number of passes in the same interspinous space $(3.32 \pm 1.44$ vs. $6.98 \pm 2.51)$ and the total time for successful lumbar puncture $(38.72 \pm 12.30 \mathrm{vs}$. $56.65 \pm 11.32 \mathrm{~s}$ ) were significantly less in Group U as compared to Group L.

Conclusion: The ultrasound guided identification of subarachanoid space was time consuming, but resulted in fewer attempts to enter the subarachnoid space when compared to the conventional landmark-based approach.
\end{abstract}

Keywords: Neuraxis, Subarachnoid space, Ultrasonography.

\section{Introduction}

Spinal anesthesia is one of the most commonly performed procedures for lower abdominal surgery.Patients with poor surface landmarks (parturients and obese) and difficult spinal anatomy such as severe lumbar scoliosis or lumbar spine surgery are the patients who pose a great technical difficulty during neuraxial blockade. ${ }^{1}$ Pre-procedural ultrasound imaging of the lumbar spine can help by providing anatomical information, permitting a more accurate estimation in identifying the lumbar intervertebral spaces, needle insertion site and the depth to the intrathecal space. ${ }^{2}$ There are numerous research stating that the use of preprocedural ultrasound has been shown to increase the first-pass success rate for spinal anesthesia in patients with difficult surface anatomic landmarks, ${ }^{3-6}$ but only limited studies had shown to improve the success rate of dural puncture when applied routinely. ${ }^{7,8}$

\section{Materials and Methods}

After obtaining institute ethics committee approval total 120 patients were recruited in the study and written informed consent obtained from them. Patients belongs to age 18 to 65 years, scheduled for surgery under spinal anaesthesia were included in this prospective randomised control trial. Patients with kyphoscoliosis, spinal deformities, previous spinal surgeries, parturients for LSCS were excluded from the study. Patients were randomised to the group L or group $\mathrm{U}$ via a computer-generated random number sequence (60 in each group). Group allocation and concealment was done by a closed envelope technique (Chart 1) 


\section{Chart 1: Consort}

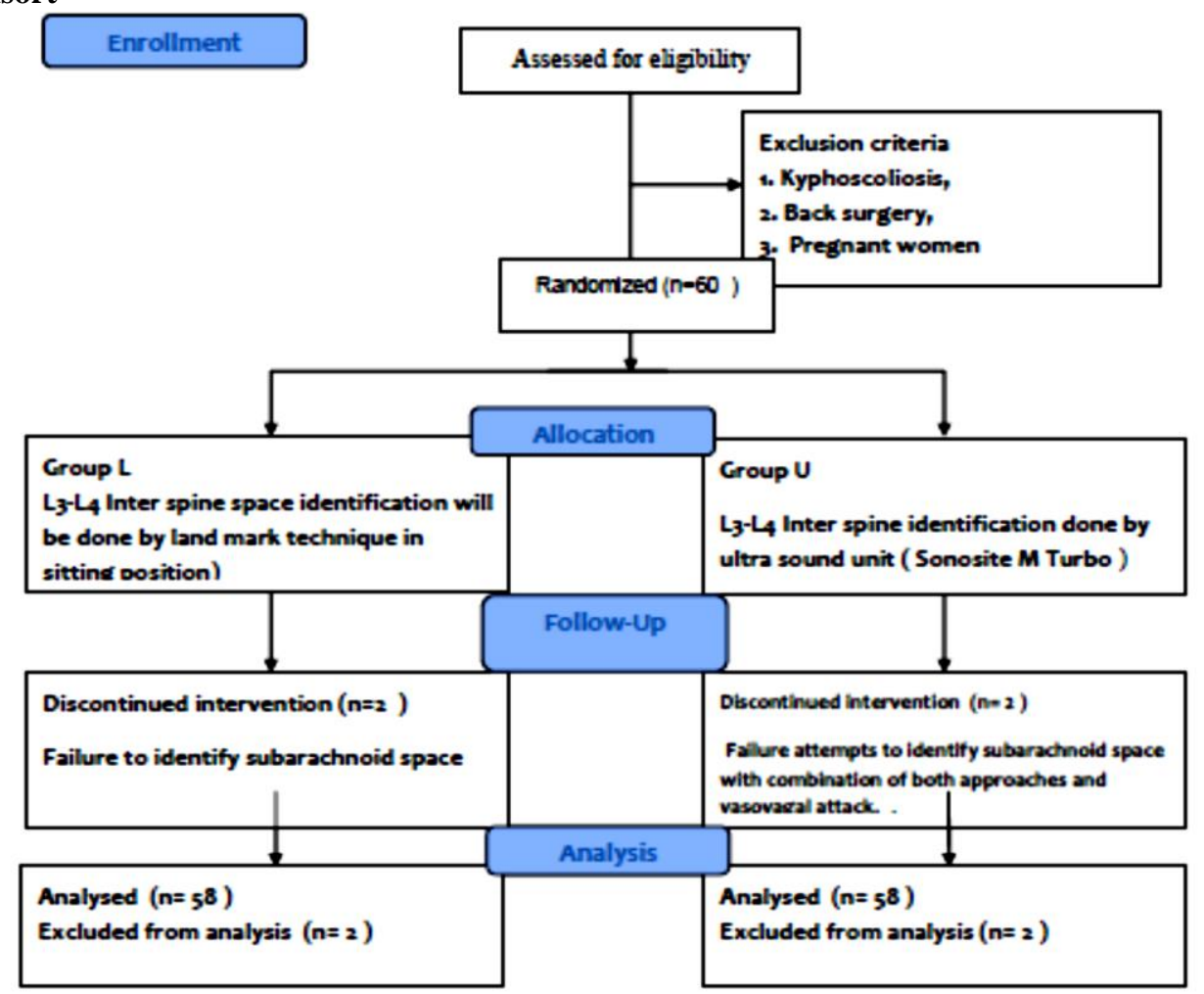

All patients were received oral pre-medication two hours before surgery, as per departmental protocol. In the operation room (OR), after attaching all standard monitors (non-invasive blood pressure, pulse oximetry and electrocardiogram) and baseline parameters recoded. Intravenous access was secured and the patients were placed in sitting posture for spinal anesthesia.

In group $\mathrm{U}$, neuraxial ultrasound was performed by anesthesiologists, who have performed more than 75 neuraxial ultrasound scans before the study. A curvilinear probe $(3-6 \mathrm{MHz})$ of portable ultrasound machine (Sonosite M Turbo ${ }^{\mathrm{TM}}$ ) was used for preprocedural marking. Ultrasound image was optimized by setting an appropriate scanning depth $(6-10 \mathrm{~cm})$, and adjusting the gain to obtain the best possible image. A paramedian sagittal oblique view of the neuraxis was obtained, and the sacrum was identified, after which the interlaminar space between L5 and S1 was noted. Subsequent interspinous spaces were identified by counting the interlaminar spaces in a cranial direction.and at the level of L3-L4 interspinous space best image of the anterior complex (anterior dura mater, posterior longitudinal ligament and body of corresponding vertebra) and posterior complex (ligamentum flavum and posterior dura mater) was obtained At this selected interspace, the probe was positioned in transverse view and a skin marker was used to mark the midpoint of the long and short borders of the probe. The point of intersection of both lines was identified as the needle entry point. ${ }^{10,11}$ (Fig. 1). Time of identification of the intersopace that is the time from which ultrasound probe is placed on the patient upto markings completed as declared by the anaesthesiologist) was noted.

In group L, L3-L4 interspace was identified by traditional landmark technique that is pont of intersection between the midline of spinous process and Tuffier's line. Time taken for the identification of the interspace (time from which the anesthesiologist started palpating to identify the landmark to completion of palpation) was noted.

Under all strict aseptic precautions, spinal anaesthesia was given with 25-G Quincke 90-mm needle at the identified interspace space. During the procedure, following outcomes were noted.

1. Insertion attempts that is defined as the number of times the spinal needle was withdrawn from the skin and reinserted)

2. Number of passes (defined as the number of advancements, that is withdrawal and redirection of the spinal needle without exiting the skin)

3. Time taken for performing the procedure (time from insertion of the needle to getting free cerebrospinal fluid $[\mathrm{CSF}]$ flow were recorded in both the groups. ${ }^{12}$

4. Presence of paraesthesia bloody tap during the procedure.

A single observer for all patients noted the outcomes. Because of the nature of the study, the observer could not be blinded to the groups. 

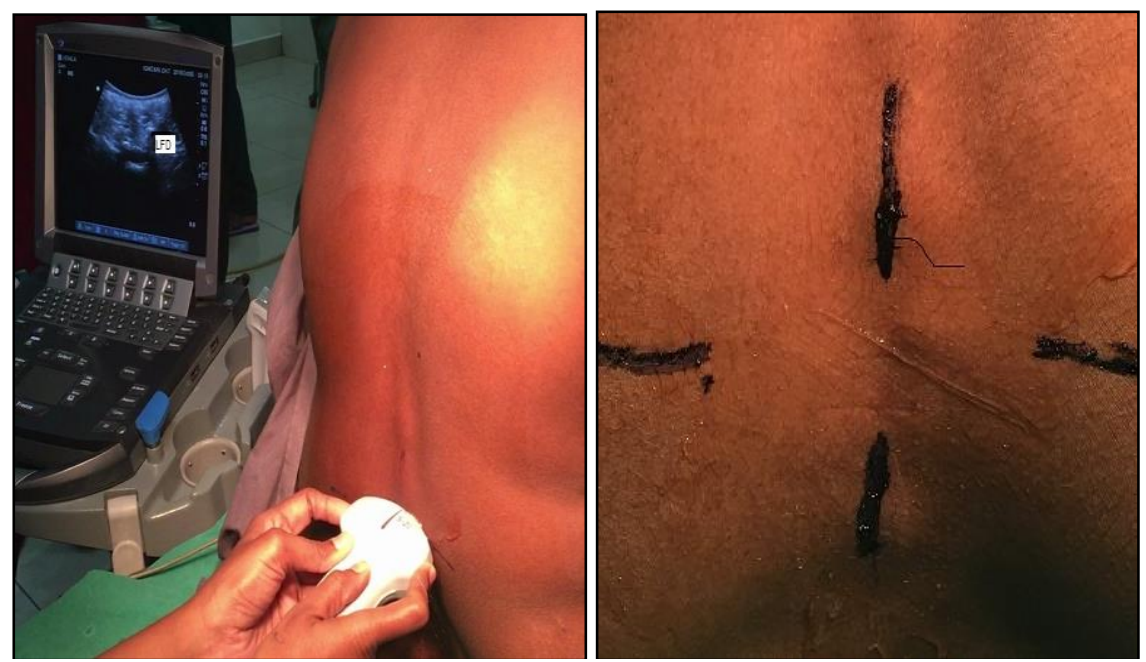

Fig. 1: A, Skin markings with the probe positioned to get the best possible transverse view of neuraxis. B, Midpoint of the long and short border of the probe marked in transverse median (TM) view. LFD = ligament flavum dura complex

The sample size was calculated using Open epi version 3, based on the study done by Karthikeyan Kallidaikurichi ${ }^{13}$ for the primary outcome, number of attempts. The average number of attempts in group $\mathrm{U}$ $(1.28 \pm 0.7)$ was approximately 0.25 times that of group $\mathrm{L}$ (mean \pm standard deviation) $(1.98 \pm 1.66)$ It was found that a total of 52 patients in each group will be needed to achieve a power of $90 \%$ and type 1 error of $<0.05$. We included 60 participants in each group to compensate for dropouts. Statistical analysis was done using SPSS software version 16 (SPSS Inc., Chicago, Illinois, USA). All data were analyzed for normal distribution using the Shapiro-Wilk test. Categorical data were analyzed using the Chi-square test or Fisher exact test as appropriate. Normally distributed parametric data were analyzed using Student t-test. All tests were two-tailed. $\mathrm{P}<0.05$ was considered statistically significant.

\section{Results}

Both the group were comparable in view of demographic profile such as age, ASA PS, BMI and types of study as shown in the Table 1 .

Two patients in group L, there was a failure to identify subarachnoid space by landmark guidance subsequent ultrasound scanning resulted in successful spinal anesthesia in these patients. One patient in group $\mathrm{U}$ received elective general anesthesia following multiple failed attempts to identify subarachnoid space with the combination of both approaches. One patient, in group $\mathrm{U}$ spinal injection, was performed in the lateral position because of a vasovagal episode.

In our study we found that the time taken for identification of interspinous space was significantly more in Group U $(56.70 \pm 13.08 \mathrm{~s})$ as compared to Group L $(47.10 \pm 10.45$ s. $)(\mathrm{P}<0.001)$ but parameters such as the number of attempts for needle insertion, number of passes in the same interspinous space and the total time for successful lumbar puncture were significantly less in Group U as compared to Group L as shown in Table 2. Successful lumbar puncture in the first attempt (Fig. 2) was significantly high in Group U $((86 \%)$ whereas in Group L $(40 \%)(\mathrm{P}<0.001)$.

Table 1: Patient characteristics in group $L$ and group $U$

\begin{tabular}{|c|c|c|c|c|}
\hline & $\begin{array}{c}\text { Group L } \\
(\mathrm{N}=58)\end{array}$ & $\begin{array}{c}\text { Group U } \\
(\mathbf{N}=58)\end{array}$ & $P$ value \\
\hline \multicolumn{2}{|l|}{ Age } & $43.56 \pm 8.979$ & $47.24 \pm 7.829$ & 0.850 \\
\hline \multirow[t]{2}{*}{ Sex } & Male & 30 & 27 & \multirow[t]{2}{*}{0.731} \\
\hline & Female & 28 & 31 & \\
\hline \multicolumn{2}{|l|}{ BMI } & $22.548 \pm 3.0195$ & $23.3779 \pm 2.3706$ & 0.241 \\
\hline \multicolumn{5}{|c|}{ Type of surgery } \\
\hline \multicolumn{2}{|c|}{ Orthopedic procedures. } & 35 & 32 & \\
\hline \multicolumn{2}{|c|}{ Hernia repair } & 8 & 11 & \\
\hline \multicolumn{2}{|c|}{ Appendicectomy } & 11 & 14 & \\
\hline \multicolumn{2}{|c|}{ Varicose vein ligation } & 4 & 2 & \\
\hline
\end{tabular}


Table 2: Comparison of space identification time, number of attempts, number of passes and total time taken for successful lumbar puncture between group $\mathrm{L}$ and group $\mathrm{U}$

\begin{tabular}{|l|c|c|c|}
\hline \multicolumn{1}{|c|}{ Parameters } & $\begin{array}{c}\text { Group L } \\
(\mathbf{n = 5 8})\end{array}$ & $\begin{array}{c}\text { Group U } \\
(\mathbf{n = 5 8})\end{array}$ & P value \\
\hline $\begin{array}{l}\text { Time for identifying subarachnoid space-time } \\
\text { (mins) }\end{array}$ & $1.12 \pm 0.52$ & $4.7 \pm 2.1$ & 0.001 \\
\hline Number of attempts for needle insertion & $1.86 \pm 0.91$ & $1.12 \pm 0.41$ & .02 \\
\hline Number of passes of the needle in the same space & $6.98 \pm 2.51$ & $3.32 \pm 1.44$ & 0.01 \\
\hline Total time for the successful lumbar puncture (s) & $56.65 \pm 11.32$ & $38.72 \pm 12.30$ & 0.041 \\
\hline
\end{tabular}

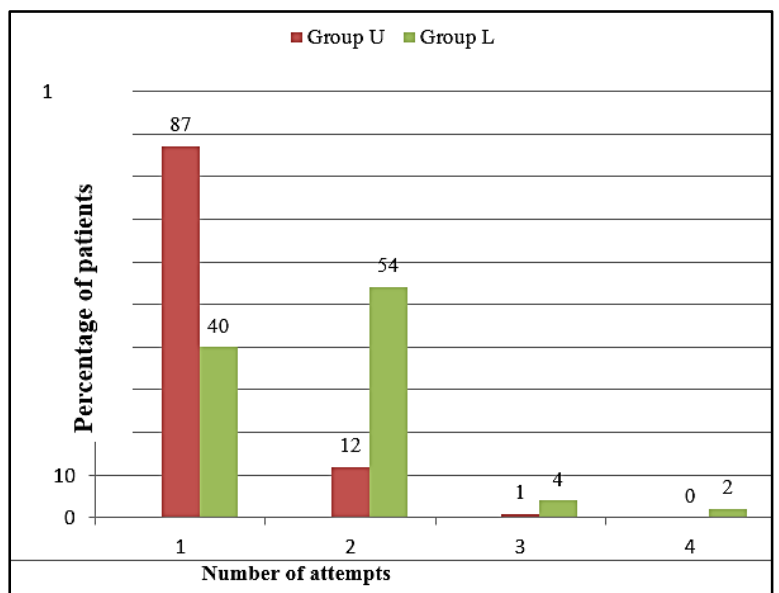

Fig. 2: Comparison of the number of attempts between group $\mathrm{L}$ and group $\mathrm{U}$

\section{Discussion}

The use of a preprocedural ultrasound assisted midline spinal technique resulted in a $>40 \%$ reduction in the number of attempts required for successful subarachnoid block compared landmark technique. Time taken for identifying the midline was longer in group P ( $4.7 \pm 2.1 \mathrm{mins})$. This was concordant with the study of Chin KJ et al, ${ }^{14,15}$ which proved that an increased time requirement to confirm anatomical landmarks in the ultrasound group (mean SD $6.7 \pm 3.1$, landmark group $0.6 \pm 0.5 \mathrm{~min}$ ), the success rate of dural puncture on the first needle insertion attempt which was twice as high in the ultrasound group as in the landmark group (65\% vs.32\%). The time consumption in identifying midline in group $U$ might be because of counting the interlaminar space was done after identifying sacrum and then proceeding in cranial direction for L3-L4 interspinous space, marking was done both in the transverse and long axis.

Neuraxial ultrasound improved first attempt success rate (86\% Vs $40 \%)$ in identifying subarachnoid space and decreased the time taken for spinal anesthesia procedures in Group U than Group C. Lim et $\mathrm{al}^{16}$ in a prospective randomized controlled trial, investigated pre-procedural ultrasonography and manual palpation in patients undergoing spinal anesthesia. And concluded the time taken for the procedure was shorter in the ultrasound group compared to manual palpation group $(2.9 \pm 3.6 \mathrm{~min}$ versus $3.9 \pm 3.7 \mathrm{~min})$ but no difference in the first-attempt success rate between the ultrasound group.

Nomura et al. ${ }^{17}$ performed a randomized study in 46 obese patients with the primary aim of determining if ultrasonography-assistance would decrease the number of lumbar puncture attempts required, in comparison to landmark palpation, in emergency department and showed that 6 of 22 attempts failed with landmark palpation but only 1 of 24 with ultrasound.

In our study, paresthesia during the procedure was significantly less in ultrasound group as compared to landmark group which can be attributed to the precise identification of interspace by ultrasound technique.

Limitations of our study include the difficulty of blinding anaesthesiologists for the study group, as the patient's group $\mathrm{U}$ had skin markings and actual needle insertion in the pre-puncture ultrasound-assisted technique remains a 'blind' procedure. More research is needed in a really challenging group of patients (obesity and spinal deformities) under real-time needle guidance.

Portability, zero radiation, real-time results and better patients satisfaction are significant advantages of ultrasound. Hence preprocedural USG should be routinely inculcated in daily anesthesia practice.

\section{Conclusion}

Preprocedural neuraxial ultrasound increases the success of lumbar puncture in first attempt with better patient satisfaction compared to traditional landmarkbased technique.

\section{Conflicts of Interest: Nil}

\section{References}

1. Myles PS, Martines RB, Bhatnagar J, de Oliveira Ramos AM, Davi HPF, Iglezias SD, et al. Ultrasound imaging facilitates spinal anesthesia in adults with difficult surface anatomic landmarks. Anesthesiol. [Internet]. 2016;20(1):376-382.

2. Manickam BP, Perlas A, Chan VWS, Brull R. The Role of a Preprocedure Systematic Sonographic Survey in Ultrasound-Guided Regional Anesthesia. Reg Anesth Pain Med. 2008;33(6):566-570.

3. Chin KJ, Perlas A, Chan V. The ultrasound-assisted paraspinous approach to lumbar neuraxial blockade: A simplified technique in patients with difficult anatomy. Acta Anaesthesiol Scand. 2015;59(5):668-673. 
4. Abd El-Hamid A, Abo Elenein G, El-fattah MHA, Elnabtity A, Hasan A. Ultrasound imaging facilitates subarachnoid blockade in patients with difficult surface anatomic landmarks. Ain-Shams J Anaesthesiol [Internet]. 2014;7(2):211.

5. Karmakar MK, Li X, Ho AMH, Kwok WH, Chui PT. Real-time ultrasound-guided paramedian epidural access: Evaluation of a novel in-plane technique. Br J Anaesth. 2009; 102(6):845-854.

6. Sahota JS, Carvalho JCA, Balki M, Fanning N, Arzola C. Ultrasound estimates for midline epidural punctures in the obese parturient: Paramedian sagittal oblique is comparable to transverse median plane. Anesth Analg. 2013;116(4):829-835.

7. Galiano K, Obwegeser AA, Bodner G, Freund M, Maurer H, Kamelger FS, et al. Ultrasound Guidance for Facet Joint Injections in the Lumbar Spine: A Computed Tomography-Controlled Feasibility Study. 2005;

8. Tran D, Kamani AA, Lessoway VA, Peterson C, Hor KW, Rohling RN. Preinsertion paramedian ultrasound guidance for epidural anesthesia. Anesth Analg. 2009; 109(2):661-667.

9. Srinivasan KK, Lee PJ, Iohom G. Ultrasound for neuraxial blockade. Med Ultrason. 2014;16(4):356-363.

10. Arzola C, Davies S. Ultrasound Using the Transverse Approach to the Lumbar Spine Provides Reliable Landmarks for Labor Epidurals. 2007;

11. R. B, T. PM. Role of ultrasonography in neuroaxial and truncal techniques. Reg Anesth Pain Med [Internet]. 2011;36(5):E79-81.

12. Tran D, Kamani AA, Al-Attas E, Lessoway VA, Massey $S$, Rohling RN. Single-operator real-time ultrasoundguidance to aim and insert a lumbar epidural needle. Can J Anesth. 2010;57(4):313-321.
13. Srinivasan KK, Iohom G, Lee PJ. Conventional Landmark-Guided Midline Versus Preprocedure Ultrasound-Guided Paramedian Techniques in Spinal Anesthesia. 2015;121(4):1089-1096.

14. Chin KJ, Perlas A, Singh M, Arzola C, Prasad A, Chan $\mathrm{V}$, et al. An ultrasound-assisted approach facilitates spinal anesthesia for total joint arthroplasty. Can J Anesth. 2009;56(9):643-650.

15. Grau T, Leipold R diger W, Horter J, Conradi R, Martin EO, Motsch J. Paramedian access to the epidural space: The optimum window for ultrasound imaging. J Clin Anesth. 2001;13(3):213-217.

16. Lim Y, Choo C, Tan K. A randomised controlled trial of ultrasound-assisted spinal anaesthesia. Anaesth Intensive Care [Internet]. 2014;42(2):191-198.

17. Nomura JT, Leech SJ, Shenbagamurthi S, Sierzenski PR, O'Connor RE, Bollinger M, et al. A randomized controlled trial of ultrasound-assisted lumbar puncture. $J$ Ultrasound Med [Internet]. 2007 Oct [cited 2018 Jun 18];26(10):1341-1348.

How to cite this article: Vaidiyanathan B, Dhanger S, Joseph R.I. Comparing the ease of identifying the subarachnoid space at midline of L3 -L4 interspace by preprocedural ultrasound versus landmark technique - A randomized control study. Indian $\mathrm{J}$ Clin Anaesth. 2018;5(4):491-495. 